\title{
On the dynamics of the mean-field polaron in the weak-coupling limit
}

\author{
Marcel Griesemer, Jochen Schmid, Guido Schneider \\ Fachbereich Mathematik, Universität Stuttgart, D-70569 Stuttgart, Germany \\ firstname.lastname@mathematik.uni-stuttgart.de
}

We consider the dynamics of the mean-field polaron in the weak-coupling limit of vanishing electron-phonon interaction, $\varepsilon \rightarrow 0$. This is a singular limit formally leading to a Schrödinger-Poisson system that is equivalent to the nonlinear Choquard equation. By establishing estimates between the approximation obtained via the Choquard equation and true solutions of the original system we show that the Choquard equation makes correct predictions about the dynamics of the polaron mean-field model for small values of $\varepsilon>0$.

\section{Introduction}

In Pekar's model of (large) polarons a single electron interacts with a dielectric polarizable elastic medium. The polarization of the medium by the charge of the electron creates an electrostatic potential that, in turn, acts on the electron. In the stationary case this leads to a self-trapped state of the electron called polaron [DA09, Lie77]. In the non-stationary case the electron triggers harmonic oscillation of the elastic medium and the combined system is described by the coupled equations

$$
\begin{aligned}
i \partial_{t} u & =-\Delta u+v u, \\
\varepsilon^{2} \partial_{t}^{2} v & =-v+\Delta^{-1}|u|^{2},
\end{aligned}
$$

for the wave function $u=u(x, t) \in \mathbb{C}$ of the electron and the electrostatic potential $v=v(x, t) \in \mathbb{R}$ associated with the polarization of the medium. Here $x \in \mathbb{R}^{3}, t \in \mathbb{R}$, and $\Delta^{-1}|u|^{2}=-(4 \pi|\cdot|)^{-1} *|u|^{2}$. The parameter $\varepsilon>0$ plays the role of the electronphonon coupling strength or the inverse of the phonon frequency in a more fundamental, quantum field theoretic model of the polaron [FG, BNAS00].

The presence of the term $\varepsilon^{2} \partial_{t}^{2} v$ in (2) leads to retardation in the self-interaction, which makes it difficult to predict the evolution of the electron. We are therefore interested 
in the question whether (11)-(2) may be solved approximately by dropping $\varepsilon^{2} \partial_{t}^{2} v$ if $\varepsilon$ is small. This approach leads to the Schrödinger-Poisson system

$$
\begin{aligned}
i \partial_{t} U & =-\Delta U+V U, \\
0 & =-V+\Delta^{-1}|U|^{2},
\end{aligned}
$$

which is equivalent to the Choquard equation

$$
i \partial_{t} U=-\Delta U+\left(\Delta^{-1}|U|^{2}\right) U
$$

Such nonlinear Hartree-type equations admit an interpretation as infinite-dimensional Hamiltonian systems, and stationary points of the associated Hamilton functionals lead to solitary-wave solutions [FTY02, MZ10]. In the present case, assuming the legitimacy of letting $\varepsilon \rightarrow 0$, solitary-wave solutions describe frictionlessly moving polarons. The Choquard equation (5) in a different context describes the evolution of coherent states (condensates) of bosons in the mean-field limit, and it has been proposed as a model for gravity-induced decoherence [EY01, Pen98]. Similar Hartree-type nonlinear equations arise in many further areas of mathematical physics.

The goal of the present paper is to prove the following approximation theorem.

Theorem 1.1. If $U \in C\left(\left[0, T_{0}\right], H^{4}\left(\mathbb{R}^{3}\right)\right)$ is a solution of (5) and $V=\Delta^{-1}|U|^{2}$, then for $C_{1}>0$ there exist $C_{2}>0$ and $\varepsilon_{0}^{\prime}>0$ such that for all $\varepsilon \in\left(0, \varepsilon_{0}^{\prime}\right]$ and all solutions $u_{\varepsilon}, v_{\varepsilon}$ of (11) and (2) satisfying

$$
\left\|u_{\varepsilon}(\cdot, 0)-U(\cdot, 0)\right\|_{H^{2}}+\left\|v_{\varepsilon}(\cdot, 0)-V(\cdot, 0)\right\|_{L^{\infty}}+\left\|\Delta\left(v_{\varepsilon}(\cdot, 0)-V(\cdot, 0)\right)\right\|_{L^{2} \cap L^{1}} \leq C_{1} \varepsilon
$$

and

we have

$$
\left\|\partial_{t} v_{\varepsilon}(\cdot, 0)\right\|_{L^{\infty}}+\left\|\Delta\left(\partial_{t} v_{\varepsilon}(\cdot, 0)\right)\right\|_{L^{2} \cap L^{1}} \leq C_{1},
$$

$$
\left\|u_{\varepsilon}(\cdot, t)-U(\cdot, t)\right\|_{H^{2}}+\left\|v_{\varepsilon}(\cdot, t)-V(\cdot, t)\right\|_{L^{\infty}}+\left\|\Delta\left(v_{\varepsilon}(\cdot, t)-V(\cdot, t)\right)\right\|_{L^{2} \cap L^{1}} \leq C_{2} \varepsilon
$$

for all $t \in\left[0, T_{0}\right]$.

See Section 4.2 for a more general version of this result - and a more precise formulation specifying, for instance, the notion of solution employed above and the spaces the solutions live in.

Remark 1.2. Such approximation results should not be taken for granted. There are various counterexamples showing that formally derived limit equations make wrong predictions about the original system [Sch95, SSZ15.

Remark 1.3. The approximation result is non-trivial since the Lipschitz constant of the right-hand side of the first-order system, cf. (18), associated to (2) is of order $\mathcal{O}\left(\varepsilon^{-1}\right)$. Hence, especially the nonlinear terms in (2) in principle can lead to some unwanted growth rates $\mathcal{O}\left(e^{\varepsilon^{-1} t}\right)$ for $t=\mathcal{O}(1)$. 
The problem described in Remark 1.3 is overcome by an integration by parts w.r.t. $t$ in the variation of constants formula associated to (2) and by a well adapted choice of spaces and norms. This allows us to use the highly oscillatory linear semigroup associated to (2) to get rid of the $\varepsilon^{-1}$ in front of the nonlinear terms, cf. also Section 5, Our estimates imply, in particular, the existence of an interval $\left[0, T_{0}\right]$, that is independent of $\varepsilon \in\left(0, \varepsilon_{0}^{\prime}\right]$, on which the system has a unique (mild) solution.

While it appears natural, mathematically, to study the limit $\varepsilon \rightarrow 0$ of (11)-(2), from a physical point of view the limit $\varepsilon \rightarrow \infty$ is even more relevant, because the system (11)(22) is believed, and partly proven, to describe the strong coupling limit, $\varepsilon \rightarrow \infty$, of the Fröhlich model of large polarons ES14, FG. We remark that the Nelson model, which is similar to the Fröhlich model, in a classical limit leads to the Schrödinger-Klein-Gordon system [AF14. The designation of (11)-(2) as mean-field polaron in the title of our paper is adopted from [BNAS00, where these equation, apparently, have been studied for the first time.

We conclude this introduction with some remarks on our notation. The intersection $X \cap Y$ and the product $X \times Y$ of two function spaces $X, Y$ on $\mathbb{R}^{3}$ will always be endowed with the sum norm $\|\cdot\|_{X \cap Y}:=\|\cdot\|_{X}+\|\cdot\|_{Y}$ and the product norm $\|(\cdot, \cdot \cdot)\|_{X \times Y}:=\|\cdot\|_{X}+$ $\|\cdot \cdot\|_{Y}$, respectively. The operator norm for bounded operators between $X$ and $Y$ will be denoted by $\|\cdot\|_{X, Y}$. We use $C_{0}\left(\mathbb{R}^{3}\right)$ to denote the space of continuous functions tending to 0 at infinity, while compact support will be indicated by the notation $C_{c}^{k}\left(\mathbb{R}^{3}\right)$ for $k \in \mathbb{N}_{0} \cup\{\infty\}$. Finally, distinct constants will be denoted with the same letter $C$ if they can be chosen independently of the small perturbation parameter $\varepsilon \ll 1$.

Acknowledgement. The work of J. Schmid is partially supported by the Deutsche Forschungsgemeinschaft DFG through the Graduiertenkolleg GRK 1838 ,,Spectral Theory and Dynamics of Quantum Systems".

\section{Spaces and operators}

In this section, we introduce the spaces we will work with and investigate the mapping properties of the Laplace operator $\Delta$ in theses spaces. In particular, we will discuss the properties of the inverse operator $\Delta^{-1}$ appearing in the equations (2) and (5). If $X, Y \subset L_{\text {loc }}^{1}\left(\mathbb{R}^{3}\right)$ are function spaces on $\mathbb{R}^{3}$, we will write $\Delta: D_{X, Y} \subset X \rightarrow Y$ to denote the linear operator $D_{X, Y} \ni u \mapsto \Delta u \in Y$ with domain

$$
D_{X, Y}:=\{u \in X: \Delta u \in Y\}
$$

where $\Delta u=\partial_{x_{1}}^{2} u+\partial_{x_{2}}^{2} u+\partial_{x_{3}}^{2} u$ denotes the distributional Laplacian of $u$. We will continually use the Sobolev spaces

$$
X_{s}:=H^{s}\left(\mathbb{R}^{3}\right) \quad \text { with } \quad\|u\|_{X_{s}}:=\left(\int\left(1+|\xi|^{2}\right)^{s}|\widehat{u}(\xi)|^{2} d \xi\right)^{1 / 2}
$$

for $s \in[0, \infty)$, whose basic and completely well-known properties are summarized in the following lemma for the sake of easy reference. 
Lemma 2.1. For $s, t \in[0, \infty)$, the following holds true:

(i) $X_{s}$ is a Hilbert space which is continuously embedded in $X_{t}$ for all $t \leq s$.

(ii) If $s \in[2, \infty)$, then $X_{s} \cdot X_{s} \subset X_{s} \cap L^{1}\left(\mathbb{R}^{3}\right)$ and there is a constant $C=C_{s}$ such that $\|u v\|_{X_{s} \cap L^{1}} \leq C\|u\|_{X_{s}}\|v\|_{X_{s}}$ for all $u, v \in X_{s}$.

(iii) $D_{X_{s}, X_{s}}=X_{s+2}$ and $\Delta: X_{s+2} \subset X_{s} \rightarrow X_{s}$ is a self-adjoint linear operator satisfying $\|\Delta u\|_{X_{s}} \leq\|u\|_{X_{s+2}}$ for all $u \in X_{s+2}$.

We will also need the following refinement of Lemma 2.1 (ii).

Lemma 2.2. If $s \in[2, \infty)$, then $X_{s-2} \cdot X_{s} \subset X_{s-2} \cap L^{1}\left(\mathbb{R}^{3}\right)$ and there is a constant $C=C_{s}$ such that

$$
\|u v\|_{X_{s-2} \cap L^{1}} \leq C\|u\|_{X_{s-2}}\|v\|_{X_{s}} \quad\left(u \in X_{s-2}, v \in X_{s}\right) .
$$

Proof. We have only to show the inclusion $X_{s-2} \cdot X_{s} \subset X_{s-2}$ and the estimate for the $X_{s-2}$-norm because the respective inclusion and estimate for $L^{1}\left(\mathbb{R}^{3}\right)$ are an immediate consequence of Schwarz's inequality. It follows from Theorem 9.3.5 in [Fri98] that for $u \in X_{s-2}$ and $v \in \mathcal{S}\left(\mathbb{R}^{3}\right)$ the product $u v$ belongs to $X_{s-2}$ with

$$
\begin{aligned}
\|u v\|_{X_{s-2}} & \leq C\|u\|_{X_{s-2}} \int\left(1+|\xi|^{2}\right)^{(s-2) / 2}|\widehat{v}(\xi)| d \xi \\
& \leq C\left(\int\left(1+|\xi|^{2}\right)^{-2} d \xi\right)^{1 / 2}\|u\|_{X_{s-2}}\|v\|_{X_{s}} \leq C\|u\|_{X_{s-2}}\|v\|_{X_{s}} .
\end{aligned}
$$

An obvious approximation argument now yields the assertion.

In the next lemma, we deal with the invertibility of $\Delta$ and the elementary properties of the inverse.

Lemma 2.3. $\Delta: D_{C_{0}, L^{2} \cap L^{1}} \subset C_{0}\left(\mathbb{R}^{3}\right) \rightarrow L^{2}\left(\mathbb{R}^{3}\right) \cap L^{1}\left(\mathbb{R}^{3}\right)$ is an invertible linear operator with full range and bounded inverse $\Delta^{-1}$ satisfying

$$
\left\|\Delta^{-1}(w)\right\|_{C_{0}} \leq C\left(\|w\|_{L^{2}}+\|w\|_{L^{1}}\right) \quad \text { and } \quad \Delta^{-1}(w)=\gamma * w
$$

for all $w \in L^{2}\left(\mathbb{R}^{3}\right) \cap L^{1}\left(\mathbb{R}^{3}\right)$, where $\gamma$ is the fundamental solution of Laplace's equation in $\mathbb{R}^{3}$ with $\gamma(x)=-1 /(4 \pi|x|)$ for $x \in \mathbb{R}^{3} \backslash\{0\}$, and where $C$ is a constant independent of $w$.

Proof. Injectivity is a simple exercise using the structure theorem for distributions with support contained in $\{0\}$. Surjectivity and the properties of $\Delta^{-1}$ are equally simple. Indeed, if $w \in L^{2}\left(\mathbb{R}^{3}\right) \cap L^{1}\left(\mathbb{R}^{3}\right)$, then

$$
\begin{aligned}
\int \frac{|\widehat{w}(\xi)|}{|\xi|^{2}} d \xi & \leq\left(\int_{|\xi|>1} \frac{1}{|\xi|^{4}} d \xi\right)^{1 / 2}\|\widehat{w}\|_{L^{2}}+\left(\int_{|\xi| \leq 1} \frac{1}{|\xi|^{2}} d \xi\right)\|\widehat{w}\|_{C_{0}} \\
& \leq C\left(\|w\|_{L^{2}}+\|w\|_{L^{1}}\right)
\end{aligned}
$$


by the continuity of the Fourier transform from $L^{1}\left(\mathbb{R}^{3}\right)$ to $C_{0}\left(\mathbb{R}^{3}\right)$. So, $\widehat{w} /|\cdot|^{2} \in L^{1}\left(\mathbb{R}^{3}\right)$ and thus $v:={ }^{\smile}\left(\widehat{w} /|\cdot|^{2}\right) \in C_{0}\left(\mathbb{R}^{3}\right)$ and, of course, $\Delta v=w$, which proves that $\Delta$ : $D_{C_{0}, L^{2} \cap L^{1}} \subset C_{0}\left(\mathbb{R}^{3}\right) \rightarrow L^{2}\left(\mathbb{R}^{3}\right) \cap L^{1}\left(\mathbb{R}^{3}\right)$ is surjective and that

$$
\Delta^{-1}(w)=-\smile\left(\widehat{w} /|\cdot|^{2}\right) \quad\left(w \in L^{2}\left(\mathbb{R}^{3}\right) \cap L^{1}\left(\mathbb{R}^{3}\right)\right) .
$$

Combining (77) and (8), we obtain the estimate in (6). Additionally, we obtain from (8) the convolution representation of $\Delta^{-1}(w)$ in (6) by virtue of the convolution theorem for tempered distributions (together with a suitable approximation argument).

In the following, $\Delta^{-1}$ will always denote the operator from the lemma above or a restriction of that operator. In order to control the nonlinear terms in (2) and (5) we use:

Lemma 2.4. If $s \in[2, \infty)$, then for all $u \in X_{s}$ and $w \in X_{s-2} \cap L^{1}\left(\mathbb{R}^{3}\right)$ one has $u \Delta^{-1}(w) \in X_{s}$ and

$$
\left\|u \Delta^{-1}(w)\right\|_{X_{s}} \leq C\|u\|_{X_{s}}\left(\|w\|_{X_{s-2}}+\|w\|_{L^{1}}\right),
$$

where $C=C_{s}$ is a constant independent of $u$ and $w$.

Proof. Clearly, we have to show the assertion only for $u \in \mathcal{S}\left(\mathbb{R}^{3}\right)$. So let $u \in \mathcal{S}\left(\mathbb{R}^{3}\right)$ and $w \in X_{s-2} \cap L^{1}\left(\mathbb{R}^{3}\right)$ and set $v:=\Delta^{-1}(w)$. Also, write

$$
\rho_{s}(\xi):=\left(1+|\xi|^{2}\right)^{s / 2} \text { and } \sigma_{s}(\xi):=|\xi|^{s}
$$

for $\xi \in \mathbb{R}^{3}$. Since $\widehat{v}=-\widehat{w} /|\cdot|^{2}$ by (8) , it follows from (7) that $\widehat{v} \in L^{1}\left(\mathbb{R}^{3}\right)$ and $\sigma_{s} \widehat{v} \in L^{2}\left(\mathbb{R}^{3}\right)$ with

$$
\|\widehat{v}\|_{L^{1}} \leq C\left(\|w\|_{X_{s-2}}+\|w\|_{L^{1}}\right) \quad \text { and } \quad\left\|\sigma_{s} \widehat{v}\right\|_{L^{2}} \leq\|w\|_{X_{s-2}}
$$

where $C$ is a constant independent of $w$ (and $s$ ). So, $\widehat{u} \in \mathcal{S}\left(\mathbb{R}^{3}\right)$ and $\widehat{v} \in L^{1}\left(\mathbb{R}^{3}\right) \subset$ $\mathcal{S}^{\prime}\left(\mathbb{R}^{3}\right)$ are classically convolvable and thus, by the convolution theorem for tempered distributions, we see that

$$
\widehat{u v}(x)=(2 \pi)^{-3 / 2}(\widehat{u} * \widehat{v})(x)=(2 \pi)^{-3 / 2} \int \widehat{u}(x-y) \widehat{v}(y) d y \quad\left(x \in \mathbb{R}^{3}\right) .
$$

Since $\rho_{s}(x) \leq C \rho_{s}(x-y)+C \sigma_{s}(y)$ for all $x, y \in \mathbb{R}^{3}$ with $C=2^{s}$, it follows that

$$
\rho_{s}(x)|\widehat{u v}(x)| \leq C\left(\left(\rho_{s}|\widehat{u}|\right) *|\widehat{v}|\right)(x)+C\left(|\widehat{u}| *\left(\sigma_{s}|\widehat{v}|\right)\right)(x)
$$

for all $x \in \mathbb{R}^{3}$ and therefore

$$
\begin{array}{r}
\left(\int\left(\rho_{s}(x)|\widehat{u v}(x)|\right)^{2} d x\right)^{1 / 2} \leq C\left\|\rho_{s}|\widehat{u}|\right\|_{L^{2}}\|\widehat{v}\|_{L^{1}}+C\|\widehat{u}\|_{L^{1}}\left\|\sigma_{s}|\widehat{v}|\right\|_{L^{2}} \\
\leq C\|u\|_{X_{s}}\left(\|w\|_{X_{s-2}}+\|w\|_{L^{1}}\right)+C\|u\|_{X_{s}}\|w\|_{X_{s-2}}
\end{array}
$$

by Young's inequality and by the inequalities (10). So, we have $u \Delta^{-1}(w) \in X_{s}$ and the estimate (9) holds true, as desired. 
In view of the above lemmas, we introduce the spaces

$$
Y_{s}:=\Delta^{-1}\left(X_{s} \cap L^{1}\left(\mathbb{R}^{3}\right)\right) \quad \text { with } \quad\|v\|_{Y_{s}}:=\|v\|_{C_{0}}+\|\Delta v\|_{X_{s}}+\|\Delta v\|_{L^{1}}
$$

for $s \in[0, \infty)$, whose basic properties are summarized in the following lemma.

Lemma 2.5. For $s, t \in[0, \infty)$, the following holds true:

(i) $Y_{s}$ is a Banach space which is continuously embedded in $Y_{t}$ for all $t \leq s$.

(ii) If $s \in[2, \infty)$, then $X_{s} \cdot Y_{s-2} \subset X_{s}$ and there is a constant $C=C_{s}$ such that $\|u v\|_{X_{s}} \leq C\|u\|_{X_{s}}\|v\|_{Y_{s-2}}$ for all $u \in X_{s}$ and $v \in Y_{s-2}$.

(iii) $\Delta^{-1}: X_{s} \cap L^{1}\left(\mathbb{R}^{3}\right) \rightarrow Y_{s}$ is a bounded linear operator.

Proof. Assertion (i) easily follows by the completeness of $C_{0}\left(\mathbb{R}^{3}\right)$ and $X_{s} \cap L^{1}\left(\mathbb{R}^{3}\right)$ and by the boundedness of $\Delta^{-1}: X_{s} \cap L^{1}\left(\mathbb{R}^{3}\right) \rightarrow C_{0}\left(\mathbb{R}^{3}\right)$ (Lemma 2.3). Assertions (ii) and (iii) are immediate consequences of Lemma 2.4 and Lemma 2.3 respectively.

\section{Solvability of the equations}

In this section, we discuss the solvability of the equations (11)-(2) and of (5), which is of course the very first thing to do in proving the desired approximation result. We start with the approximation equation (5) and first show mild and classical solvability of the corresponding abstract initial value problem

$$
U^{\prime}=i \Delta U-i U \Delta^{-1}\left(|U|^{2}\right), \quad \text { with } \quad U(0)=U_{0}
$$

in the sense of $[\mathrm{Paz} 83]$.

Theorem 3.1. If $s \in[0, \infty)$, then for every $U_{0} \in X_{s+2}$ there exists a $T_{0}>0$ and $a$ unique mild solution $U \in C\left(I, X_{s+2}\right)$ of (13) on $I=\left[0, T_{0}\right]$.

Proof. Clearly, the linear part $i \Delta$ of the equation (13) is the generator of a strongly continuous unitary group in $X_{s+2}$ by virtue of Lemma 2.1 (iii). Also, the nonlinear part $f$ of the equation (13) given by

$$
f(U):=-i U \Delta^{-1}\left(|U|^{2}\right)
$$

is a map from $X_{s+2}$ into itself and Lipschitz continuous on bounded subsets by virtue of Lemma 2.5 (ii) and (iii). So, the standard existence and uniqueness result for mild solutions (Theorem 6.1.4 in [Paz83]) implies that there is a $T_{0}>0$ and a unique mild solution $U: I=\left[0, T_{0}\right] \rightarrow X_{s+2}$ of (13). In other words, the integral equation

$$
U(t)=e^{i \Delta t} U_{0}-i \int_{0}^{t} e^{i \Delta(t-r)} U(r) \Delta^{-1}\left(|U(r)|^{2}\right) d r
$$

has a unique solution $U \in C\left(I, X_{s+2}\right)$. 
In the situation of the above theorem, we also obtain classical solvability by Theorem 6.1.5 in [Paz83]:

Corollary 3.2. If $s \in[0, \infty)$ and if $U_{0} \in X_{s+2}$ and $U \in C\left(I, X_{s+2}\right)$ are as in the above theorem, then $U$ belongs to $C^{1}\left(I, X_{s}\right)$ and is a classical solution of (13).

We now go on with the original equations (11) and (2) and show mild and classical solvability of the corresponding abstract initial value problem

$$
\left(\begin{array}{c}
u \\
v \\
w
\end{array}\right)^{\prime}=\left(\begin{array}{c}
i \Delta u \\
\varepsilon^{-1} w \\
-\varepsilon^{-1} v
\end{array}\right)+\left(\begin{array}{c}
-i u v \\
0 \\
\varepsilon^{-1} \Delta^{-1}\left(|u|^{2}\right)
\end{array}\right), \quad \text { with } \quad\left(\begin{array}{c}
u \\
v \\
w
\end{array}\right)(0)=\left(\begin{array}{c}
u_{0} \\
v_{0} \\
w_{0}
\end{array}\right)
$$

in the sense of Paz83]. In the following, we will always abbreviate

$$
\Lambda(v, w):=(w,-v) \quad \text { for } \quad(v, w) \in Y_{s} \times Y_{s} .
$$

Lemma 3.3. If $s \in[0, \infty)$, then $\varepsilon^{-1} \Lambda$ is the generator of a continuous group $\left(e^{\varepsilon^{-1} \Lambda t}\right)_{t \in \mathbb{R}}$ in $Y_{s} \times Y_{s}$, which is uniformly bounded w.r.t. $\varepsilon \in(0, \infty)$, that is,

$$
\sup _{\varepsilon \in(0, \infty), t \in \mathbb{R}}\left\|e^{\varepsilon^{-1} \Lambda t}\right\|_{Y_{s} \times Y_{s}, Y_{s} \times Y_{s}}<\infty .
$$

Proof. Since $\Lambda$ is a bounded operator in $Y_{s} \times Y_{s}$ having the matrix representation

$$
\Lambda=\left(\begin{array}{cc}
0 & 1 \\
-1 & 0
\end{array}\right)
$$

we have the explicit representation formula

$$
e^{\varepsilon^{-1} \Lambda t}=\sum_{n=0}^{\infty} \frac{\left(\varepsilon^{-1} \Lambda t\right)^{n}}{n !}=\left(\begin{array}{cc}
\cos \left(\varepsilon^{-1} t\right) & \sin \left(\varepsilon^{-1} t\right) \\
-\sin \left(\varepsilon^{-1} t\right) & \cos \left(\varepsilon^{-1} t\right)
\end{array}\right)
$$

from which the assertion is obvious.

Theorem 3.4. If $s \in[2, \infty)$ and $\varepsilon>0$, then for every $\left(u_{0}, v_{0}, w_{0}\right)=\left(u_{0 \varepsilon}, v_{0 \varepsilon}, w_{0 \varepsilon}\right) \in$ $X_{s} \times Y_{s-2} \times Y_{s-2}$ there exists a unique maximal mild solution $(u, v, w)=\left(u_{\varepsilon}, v_{\varepsilon}, w_{\varepsilon}\right) \in$ $C\left(I_{\varepsilon}, X_{s} \times Y_{s-2} \times Y_{s-2}\right)$ of (15) with $I_{\varepsilon} \subset I$, where $I=\left[0, T_{0}\right]$ is the interval from the previous theorem.

Proof. Clearly, the linear part $A=A_{\varepsilon}=\operatorname{diag}\left(i \Delta, \varepsilon^{-1} \Lambda\right)$ of the equation (15) given by

$$
\begin{gathered}
A(u, v, w):=\left(i \Delta u, \varepsilon^{-1} \Lambda(v, w)\right)=\left(i \Delta u, \varepsilon^{-1} w,-\varepsilon^{-1} v\right) \\
\left((u, v, w) \in X_{s+2} \times Y_{s-2} \times Y_{s-2}\right)
\end{gathered}
$$


is the generator of a strongly continuous group $\left(e^{A_{\varepsilon} t}\right)_{t \in \mathbb{R}}=\left(\operatorname{diag}\left(e^{i \Delta t}, e^{\varepsilon^{-1} \Lambda t}\right)\right)_{t \in \mathbb{R}}$ in $X_{s} \times Y_{s-2} \times Y_{s-2}$ by virtue of Lemma 2.1 (iii) and Lemma 3.3. Also, the nonlinear part $f=f_{\varepsilon}$ of the equation (15) given by

$$
f(u, v, w):=\left(-i u v, 0, \varepsilon^{-1} \Delta^{-1}\left(|u|^{2}\right)\right)
$$

is a map from $X_{s} \times Y_{s-2} \times Y_{s-2}$ into itself and Lipschitz on bounded subsets by virtue of Lemma 2.5 (ii)-(iii) and Lemma 2.1 (ii). So, for $I=\left[0, T_{0}\right]$ as in Theorem 3.1. the standard existence and uniqueness result for mild solutions (Theorem 6.1.4 in [Paz83]) implies that there is a unique maximal mild solution $\left(u_{\varepsilon}, v_{\varepsilon}, w_{\varepsilon}\right): I_{\varepsilon} \rightarrow X_{s} \times Y_{s-2} \times Y_{s-2}$ of (15) with $I_{\varepsilon} \subset I$. In other words, the integral equation

$$
\left(\begin{array}{c}
u \\
v \\
w
\end{array}\right)(t)=e^{A_{\varepsilon} t}\left(\begin{array}{c}
u_{0} \\
v_{0} \\
w_{0}
\end{array}\right)+\int_{0}^{t} e^{A_{\varepsilon}(t-r)}\left(\begin{array}{c}
-i u(r) v(r) \\
0 \\
\varepsilon^{-1} \Delta^{-1}\left(|u(r)|^{2}\right)
\end{array}\right) d r
$$

has a unique maximal solution $(u, v, w)=\left(u_{\varepsilon}, v_{\varepsilon}, w_{\varepsilon}\right) \in C\left(I_{\varepsilon}, X_{s} \times Y_{s-2} \times Y_{s-2}\right)$ with $I_{\varepsilon} \subset I$.

In the situation of the above theorem, we also obtain classical solvability by Theorem 6.1.5 in [Paz83]:

Corollary 3.5. If $s \in[2, \infty)$ and $\varepsilon>0$ and if $\left(u_{0}, v_{0}, w_{0}\right)$ and $(u, v, w)=\left(u_{\varepsilon}, v_{\varepsilon}, w_{\varepsilon}\right) \in$ $C\left(I_{\varepsilon}, X_{s} \times Y_{s-2} \times Y_{s-2}\right)$ are as in the above theorem, then $(u, v, w)$ belongs to $C^{1}\left(I_{\varepsilon}, X_{s-2} \times\right.$ $\left.Y_{s-2} \times Y_{s-2}\right)$ and is a classical solution of (15).

For the subsequent estimates, we additionally have to control the second-order time derivatives.

Lemma 3.6. Suppose $U \in C\left(I, X_{s+2}\right)$ is as in Theorem 3.1 with $s \in[2, \infty)$ and $V(t):=$ $\Delta^{-1}\left(|U(t)|^{2}\right)$ for $t \in I$. Suppose further $(u, v, w)=\left(u_{\varepsilon}, v_{\varepsilon}, w_{\varepsilon}\right) \in C\left(I_{\varepsilon}, X_{s} \times Y_{s-2} \times Y_{s-2}\right)$ is as in Theorem 3.4 with the same $s \in[2, \infty)$ as above. Then $V \in C^{2}\left(I, Y_{s-2}\right)$ and $v \in C^{2}\left(I_{\varepsilon}, Y_{s-2}\right)$.

Proof. With the help of Lemma 2.1 (ii) and (iii) and of Lemma 2.5 (ii) and (iii), it follows from Corollary 3.2 and (13) that $U^{\prime} \in C^{1}\left(I, X_{s-2}\right)$ and hence $U \in C^{1}\left(I, X_{s}\right) \cap$ $C^{2}\left(I, X_{s-2}\right)$. We easily conclude from this by Lemma 2.2 that $|U|^{2} \in C^{2}\left(I, X_{s-2}\right)$ and therefore $V=\Delta^{-1}\left(|U|^{2}\right)$ belongs to $C^{2}\left(I, Y_{s-2}\right)$ by Lemma 2.5 (iii). That $v$ belongs to $C^{2}\left(I_{\varepsilon}, Y_{s-2}\right)$ is an immediate consequence of Corollary 3.5 and (15).

\section{Approximation error and approximation result}

In this section, we are going to bound the approximation error, that is the difference between the solutions $u=u_{\varepsilon}, v=v_{\varepsilon}$ of the original equations (11)-(2) and the solutions $U, V$ of the approximate equations (3)-(4). We show that this difference - measured in the right norm - remains of order $\varepsilon$ for all times $t \in\left[0, T_{0}\right]$ provided it was of order $\varepsilon$ at the initial time 0 , thus establishing the desired approximation result. 


\subsection{Integral equations and estimates for the error}

We first derive integral equations for the scaled approximation errors

$$
\begin{gathered}
R_{u}(t)=R_{u, \varepsilon}(t):=\varepsilon^{-1}(u(t)-U(t)), \quad R_{v}(t)=R_{v, \varepsilon}(t):=\varepsilon^{-1}(v(t)-V(t)), \\
R_{w}(t)=R_{w, \varepsilon}(t):=\varepsilon R_{v}^{\prime}(t)=v^{\prime}(t)-V^{\prime}(t),
\end{gathered}
$$

where $U \in C\left(I, X_{s+2}\right)$ and $(u, v, w)=\left(u_{\varepsilon}, v_{\varepsilon}, w_{\varepsilon}\right) \in C\left(I_{\varepsilon}, X_{s} \times Y_{s-2} \times Y_{s-2}\right)$ are mild solutions of (13) and (15) with $s \in[2, \infty)$ and where $V=\Delta^{-1}\left(|U|^{2}\right)$. With the help of Corollary 3.2 and 3.5 and Lemma 3.6 . we obtain

$$
R_{u}(t)=e^{i \Delta t} R_{u}(0)-i \int_{0}^{t} e^{i \Delta(t-r)} f_{u}(r) d r
$$

for all $t \in I_{\varepsilon}$, where

$$
f_{u}(r)=f_{u, \varepsilon}(r):=R_{u}(r) V(r)+U(r) R_{v}(r)+\varepsilon R_{u}(r) R_{v}(r) .
$$

and

$$
\left(\begin{array}{l}
R_{v}(t) \\
R_{w}(t)
\end{array}\right)=e^{\varepsilon^{-1} \Lambda t}\left(\begin{array}{l}
R_{v}(0) \\
R_{w}(0)
\end{array}\right)+\int_{0}^{t} e^{\varepsilon^{-1} \Lambda(t-r)}\left(\begin{array}{c}
0 \\
\varepsilon^{-1} f_{v}(r)-V^{\prime \prime}(r)
\end{array}\right) d r
$$

for all $t \in I_{\varepsilon}$, where

$$
f_{v}(r)=f_{v, \varepsilon}(r):=\Delta^{-1}\left(R_{u}(r) \overline{U(r)}+\overline{R_{u}(r)} U(r)+\varepsilon\left|R_{u}(r)\right|^{2}\right) .
$$

We now derive from the integral equations (17) and (18) integral inequalities which are implicit in the sense that the scaled approximation errors $R_{u}$ and $\left(R_{v}, R_{w}\right)$ - measured in the norm of $X_{s}$ and $Y_{s-2} \times Y_{s-2}$ respectively - show up on both sides of the inequalities. In order to get rid of the dangerous $\varepsilon^{-1}$ in front of $f_{v}$ in (18) we perform an integration by parts.

Proposition 4.1. Set

$$
S_{u, \varepsilon}(t):=\sup _{r \in[0, t]}\left\|R_{u}(r)\right\|_{X_{s}}, \quad S_{(v, w), \varepsilon}(t):=\sup _{r \in[0, t]}\left\|\left(R_{v}(r), R_{w}(r)\right)\right\|_{Y_{s-2} \times Y_{s-2}},
$$

and $S_{\varepsilon}(t):=S_{u, \varepsilon}(t)+S_{(v, w), \varepsilon}(t)$ for $t \in I_{\varepsilon}$. Then there is a constant $C=C_{s}$ such that for all $\varepsilon \in(0, \infty)$ and all $t \in I_{\varepsilon}$

$$
\begin{aligned}
S_{u, \varepsilon}(t) & \leq C\left(S_{\varepsilon}(0)+\int_{0}^{t} S_{\varepsilon}(r)+\varepsilon S_{\varepsilon}(r)^{2} d r\right), \\
S_{(v, w), \varepsilon}(t) & \leq C\left(S_{\varepsilon}(0)+1+S_{u, \varepsilon}(t)+\varepsilon S_{u, \varepsilon}(t)^{2}+\int_{0}^{t} S_{\varepsilon}(r)+\varepsilon S_{\varepsilon}(r)^{2}+\varepsilon^{2} S_{\varepsilon}(r)^{3} d r\right) .
\end{aligned}
$$


Proof. It follows from Lemma 2.5 (ii) that $f_{u}$ belongs to $C\left(I_{\varepsilon}, X_{s}\right)$ and satisfies the estimate

$$
\begin{gathered}
\left\|f_{u}(r)\right\|_{X_{s}} \leq C\left(\left\|R_{u}(r)\right\|_{X_{s}}\|V(r)\|_{Y_{s-2}}+\|U(r)\|_{X_{s}}\left\|R_{v}(r)\right\|_{Y_{s-2}}\right. \\
\left.+\varepsilon\left\|R_{u}(r)\right\|_{X_{s}}\left\|R_{v}(r)\right\|_{Y_{s-2}}\right)
\end{gathered}
$$

for all $r \in I_{\varepsilon}$. Since $\sup _{r \in I}\|U(r)\|_{X_{s}}<\infty$ and $\sup _{r \in I}\|V(r)\|_{Y_{s-2}}<\infty$, the asserted estimate for $S_{u, \varepsilon}$ now follows from (17) with the help of (19) and Lemma 2.1 (iii). Since $R_{u} \in C\left(I_{\varepsilon}, X_{s}\right) \cap C^{1}\left(I_{\varepsilon}, X_{s-2}\right)$ and $U \in C^{1}\left(I, X_{s}\right)$ by Corollary 3.2 and 3.5, it follows from Lemma 2.5 (iii) and Lemma 2.2 that $f_{v}$ belongs to $C^{1}\left(I_{\varepsilon}, Y_{s-2}\right)$. We can therefore integrate by parts in (18) and thus obtain

$$
\begin{aligned}
\left(\begin{array}{c}
R_{v}(t) \\
R_{w}(t)
\end{array}\right)= & e^{\varepsilon^{-1} \Lambda t}\left(\begin{array}{c}
R_{v}(0) \\
R_{w}(0)
\end{array}\right)-\int_{0}^{t} e^{\varepsilon^{-1} \Lambda(t-r)}\left(\begin{array}{c}
0 \\
V^{\prime \prime}(r)
\end{array}\right) d r \\
& -\left.e^{\varepsilon^{-1} \Lambda(t-r)} \Lambda^{-1}\left(\begin{array}{c}
0 \\
f_{v}(r)
\end{array}\right)\right|_{r=0} ^{r=t}+\int_{0}^{t} e^{\varepsilon^{-1} \Lambda(t-r)} \Lambda^{-1}\left(\begin{array}{c}
0 \\
f_{v}^{\prime}(r)
\end{array}\right) d r
\end{aligned}
$$

for all $t \in I_{\varepsilon}$, where

$$
\begin{aligned}
f_{v}^{\prime}(r) & =\Delta^{-1}\left(R_{u}(r) \overline{U^{\prime}(r)}+R_{u}^{\prime}(r)\left(\overline{U(r)}+\varepsilon \overline{R_{u}(r)}\right)\right)+\text { c.c. } \\
& =\Delta^{-1}\left(R_{u}(r) \overline{U^{\prime}(r)}\right)+i \Delta^{-1}\left(\left(\Delta R_{u}(r)-f_{u}(r)\right)\left(\overline{U(r)}+\varepsilon \overline{R_{u}(r)}\right)\right)+\text { c.c.. }
\end{aligned}
$$

In the above equation, the symbol c.c. stands for the complex conjugate of the terms on the left of it. With the help of Lemma 2.5 (iii) and Lemma 2.1 (ii), we can estimate

$$
\begin{aligned}
\left\|f_{v}(r)\right\|_{Y_{s-2}} & \leq C\left(\left\|R_{u}(r) \overline{U(r)}\right\|_{X_{s} \cap L^{1}}+\varepsilon\left\|R_{u}(r) \overline{R_{u}(r)}\right\|_{X_{s} \cap L^{1}}\right) \\
& \leq C\left(\left\|R_{u}(r)\right\|_{X_{s}}\|U(r)\|_{X_{s}}+\varepsilon\left\|R_{u}(r)\right\|_{X_{s}}^{2}\right)
\end{aligned}
$$

for all $r \in I_{\varepsilon}$, and with the help of Lemma 2.5 (iii) and Lemmas 2.2 and 2.1 (ii)-(iii), we can estimate

$$
\begin{gathered}
\left\|f_{v}^{\prime}(r)\right\|_{Y_{s-2}} \leq C\left(\left\|R_{u}(r) \overline{U^{\prime}(r)}\right\|_{X_{s} \cap L^{1}}+\left\|\left(\Delta R_{u}(r)\right)\left(\overline{U(r)}+\varepsilon \overline{R_{u}(r)}\right)\right\|_{X_{s-2} \cap L^{1}}\right. \\
\left.\left.+\| f_{u}(r) \overline{(U(r)}+\varepsilon \overline{R_{u}(r)}\right) \|_{X_{s} \cap L^{1}}\right) \\
\leq C\left(\left\|R_{u}(r)\right\|_{X_{s}}\left\|U^{\prime}(r)\right\|_{X_{s}}+\left\|R_{u}(r)\right\|_{X_{s}}\left(\|U(r)\|_{X_{s}}+\varepsilon\left\|R_{u}(r)\right\|_{X_{s}}\right)\right. \\
\left.+\left\|f_{u}(r)\right\|_{X_{s}}\left(\|U(r)\|_{X_{s}}+\varepsilon\left\|R_{u}(r)\right\|_{X_{s}}\right)\right)
\end{gathered}
$$

for all $r \in I_{\varepsilon}$. Since $\sup _{r \in I}\|U(r)\|_{X_{s}}+\left\|U^{\prime}(r)\right\|_{X_{s}}<\infty$ and $\sup _{r \in I}\|V(r)\|_{Y_{s-2}}+$ $\left\|V^{\prime \prime}(r)\right\|_{Y_{s-2}}<\infty$, the asserted estimate for $S_{(v, w), \varepsilon}$ now follows from (20) with the help of (21), (22), (19) and Lemma 3.3. 


\subsection{Approximation result}

With the help of Gronwall's lemma, we finally turn the implicit estimates for the approximation error just established into explicit estimates and thus obtain our approximation theorem. Choosing $s=2$, we obtain the version of the theorem stated in the introduction.

Theorem 4.2. Suppose $(U, V)$ and $(u, v, w)=\left(u_{\varepsilon}, v_{\varepsilon}, w_{\varepsilon}\right)$ are as in Lemma 3.6 and suppose further that the initial values satisfy

$$
\left\|u_{\varepsilon}(0)-U(0)\right\|_{X_{s}}+\left\|v_{\varepsilon}(0)-V(0)\right\|_{Y_{s-2}}+\varepsilon\left\|v_{\varepsilon}^{\prime}(0)-V^{\prime}(0)\right\|_{Y_{s-2}} \leq C_{1} \varepsilon
$$

for all $\varepsilon \in\left(0, \varepsilon_{0}\right]$ with some $\varepsilon_{0}>0$ and some constant $C_{1}=C_{1, s}$. Then there is an $\varepsilon_{0}^{\prime} \in\left(0, \varepsilon_{0}\right]$ and a constant $C_{2}=C_{2, s}$ such that $I_{\varepsilon}=I$ for all $\varepsilon \in\left(0, \varepsilon_{0}^{\prime}\right]$ and such that

$$
\left\|u_{\varepsilon}(t)-U(t)\right\|_{X_{s}}+\left\|v_{\varepsilon}(t)-V(t)\right\|_{Y_{s-2}}+\varepsilon\left\|v_{\varepsilon}^{\prime}(t)-V^{\prime}(t)\right\|_{Y_{s-2}} \leq C_{2} \varepsilon
$$

for all $t \in I$ and all $\varepsilon \in\left(0, \varepsilon_{0}^{\prime}\right]$.

Proof. We plug in the estimate for $S_{u, \varepsilon}$ into the estimate for $S_{(v, w), \varepsilon}$ from Proposition 4.1 and, by adding the resulting inequality to the inequality for $S_{u, \varepsilon}$, we obtain the following inequality for $S_{\varepsilon}$ :

$$
S_{\varepsilon}(t) \leq C\left(S_{\varepsilon}(0)+1+\int_{0}^{t} S_{\varepsilon}(r)+\varepsilon S_{\varepsilon}(r)^{2}+\varepsilon^{2} S_{\varepsilon}(r)^{3}+\varepsilon^{3} S_{\varepsilon}(r)^{4} d r\right)
$$

for all $t \in I_{\varepsilon}$ and all $\varepsilon \in(0, \infty)$. Since $S_{\varepsilon}(0) \leq C_{1}$ for all $\varepsilon \in\left(0, \varepsilon_{0}\right]$ by assumption, we therefore have that

$$
S_{\varepsilon}(t) \leq C+C \int_{0}^{t} p\left(\varepsilon S_{\varepsilon}(r)\right) S_{\varepsilon}(r) d r
$$

for all $t \in I_{\varepsilon}$ and all $\varepsilon \in\left(0, \varepsilon_{0}\right]$, where $p(\xi):=1+\xi+\xi^{2}+\xi^{3}$ for $\xi \in \mathbb{R}^{3}$. So, by Gronwall's lemma, we obtain

$$
S_{\varepsilon}(t) \leq C e^{C \int_{0}^{t} p\left(\varepsilon S_{\varepsilon}(r)\right) d r}
$$

for all $t \in I_{\varepsilon}$ and all $\varepsilon \in\left(0, \varepsilon_{0}\right]$. Set $M:=C e^{T_{0}}+2$ with $C$ being the constant in (26) and choose $\varepsilon_{0}^{\prime} \in\left(0, \varepsilon_{0}\right]$ such that

$$
C e^{p\left(\varepsilon_{0}^{\prime} M\right) T_{0}} \leq C e^{T_{0}}+1=M-1 .
$$

Also, for $\varepsilon \in\left(0, \varepsilon_{0}^{\prime}\right]$ set

$$
b_{\varepsilon}:=\sup \left\{t \in I_{\varepsilon}: S_{\varepsilon}(r) \leq M \text { for all } r \in[0, t]\right\} .
$$

We then have, for all $t \in\left[0, b_{\varepsilon}\right)$ and all $\varepsilon \in\left(0, \varepsilon_{0}^{\prime}\right]$, that

$$
S_{\varepsilon}(t) \leq C e^{C \int_{0}^{t} p\left(\varepsilon S_{\varepsilon}(r)\right) d r} \leq C e^{p\left(\varepsilon_{0}^{\prime} M\right) T_{0}} \leq M-1
$$


by virtue of (26) and (27). If now $b_{\varepsilon}$ was strictly less than $\sup I_{\varepsilon}$ for some $\varepsilon \in\left(0, \varepsilon_{0}^{\prime}\right]$, then from (28) we would obtain, using the continuity of $S_{\varepsilon}$, a contradiction to the definition of $b_{\varepsilon}$. So, $b_{\varepsilon}=\sup I_{\varepsilon}$ for all $\varepsilon \in\left(0, \varepsilon_{0}^{\prime}\right]$. It follows from this and from (28) that

$$
\sup _{t \in I_{\varepsilon}} S_{\varepsilon}(t)=\sup _{t \in\left[0, b_{\varepsilon}\right)} S_{\varepsilon}(t) \leq M-1
$$

and hence

$$
\sup _{t \in I_{\varepsilon}}\left\|\left(u_{\varepsilon}(t), v_{\varepsilon}(t), w_{\varepsilon}(t)\right)\right\|_{X_{s} \times Y_{s-2} \times Y_{s-2}} \leq C+\varepsilon_{0}^{\prime} \sup _{t \in I_{\varepsilon}} S_{\varepsilon}(t)<\infty
$$

for all $\varepsilon \in\left(0, \varepsilon_{0}^{\prime}\right]$. Since $\left(u_{\varepsilon}, v_{\varepsilon}, w_{\varepsilon}\right)$ by definition is the maximal mild solution of (15) with $I_{\varepsilon} \subset I$, it follows from (30) by the standard blow-up result for mild solutions (Theorem 6.1.4 in Paz83]) that $I_{\varepsilon}$ must be equal to $I$ for all $\varepsilon \in\left(0, \varepsilon_{0}^{\prime}\right]$. So, invoking (29) again, we see that

$$
\sup _{t \in I} S_{\varepsilon}(t)=\sup _{t \in I_{\varepsilon}} S_{\varepsilon}(t) \leq M-1=: C_{2}
$$

for all $\varepsilon \in\left(0, \varepsilon_{0}^{\prime}\right]$, and this immediately implies (24) .

\section{Concluding remarks}

We close this paper with some remarks on the connection of the presented approach to normal form transformations. Instead of the approach pursued above, one can try to get rid of the dangerous term $\varepsilon^{-1} \Delta^{-1}\left(U \overline{R_{u}}+\bar{U} R_{u}\right)$ in the equation (18) for $R_{(v, w)}:=$ $\left(R_{v}, R_{w}\right)$ by a near-identity change of coordinates of the form

$$
\widetilde{R}_{(v, w)}=R_{(v, w)}+B\left(U, R_{u}\right)
$$

where $B$ is a symmetric bilinear mapping. Inserting this transformation into the $R_{(v, w)}$ equation yields

$$
\begin{aligned}
\partial_{t} \widetilde{R}_{(v, w)}= & \varepsilon^{-1} \Lambda \widetilde{R}_{(v, w)}-\varepsilon^{-1} \Lambda B\left(U, R_{u}\right)+B\left(-i \Delta U, R_{u}\right)+B\left(U,-i \Delta R_{u}\right) \\
& +\varepsilon^{-1} \Delta^{-1}\left(U \overline{R_{u}}+\bar{U} R_{u}\right)+\text { h.o.t., }
\end{aligned}
$$

where h.o.t. stands for the higher-order terms. So, we have to find a bilinear mapping $B$ such that

$$
-\varepsilon^{-1} \Lambda B\left(U, R_{u}\right)+B\left(-i \Delta U, R_{u}\right)+B\left(U,-i \Delta R_{u}\right)+\varepsilon^{-1} \Delta^{-1}\left(U \overline{R_{u}}+\bar{U} R_{u}\right)=0,
$$

which is not possible, however, since the non-resonance condition

$$
\inf _{k, l}\left| \pm \varepsilon^{-1}+(k-l)^{2}-l^{2}\right|>0
$$


is not satisfied. The approach we took above corresponds to the choice $B\left(U, R_{u}\right)=$ $\Lambda^{-1} \Delta^{-1}\left(U \overline{R_{u}}+\bar{U} R_{u}\right)=\mathcal{O}(1)$. It allowed us to eliminate the terms of order $\mathcal{O}\left(\varepsilon^{-1}\right)$ such that after the transform we had

$$
\begin{aligned}
\partial_{t} \widetilde{R}_{(v, w)} & =\varepsilon^{-1} \Lambda \widetilde{R}_{(v, w)}+B\left(-i \Delta U, R_{u}\right)+B\left(U,-i \Delta R_{u}\right)+\cdots \\
& =\varepsilon^{-1} \Lambda \widetilde{R}_{(v, w)}+\mathcal{O}(1) .
\end{aligned}
$$

We finally remark that the energy approach chosen in DSS16] for a similar limit in the Klein-Gordon-Zakharov system can only be used for (12)-(2) in space dimensions $d \geq 5$ due to the occurrence of $\Delta^{-1}$ which maps $L^{1}\left(\mathbb{R}^{d}\right) \cap L^{2}\left(\mathbb{R}^{d}\right)$ into $L^{2}\left(\mathbb{R}^{d}\right)$ only for space dimensions $d \geq 5$.

\section{References}

[AF14] Zied Ammari and Marco Falconi. Wigner measures approach to the classical limit of the Nelson model: convergence of dynamics and ground state energy. J. Stat. Phys., 157(2):330-362, 2014.

[BNAS00] P. Bechouche, J. Nieto, E. Ruiz Arriola, and J. Soler. On the time evolution of the mean-field polaron. Journal of Mathematical Physics, 41(7):4293-4312, 2000 .

[DA09] J.T. Devreese and A.S. Alexandrov. Fröhlich polaron and bipolaron: recent developments. Reports on Progress in Physics, 72(6):066501, 2009.

[DSS16] M. Daub, G. Schneider, and K. Schratz. From the Klein-Gordon-Zakharov system to the Klein-Gordon equation. Math. Meth. Appl. Sciences, accepted, 2016.

[EY01] László Erdős and Horng-Tzer Yau. Derivation of the nonlinear Schrödinger equation from a many body Coulomb system. Adv. Theor. Math. Phys., 5(6):1169-1205, 2001.

[FG] R.L. Frank and Z. Gang. Derivation of an effective evolution equation for a strongly coupled polaron. arXiv:1505.03059.

[Fri98] F. G. Friedlander. Introduction to the theory of distributions. Cambridge University Press, Cambridge, second edition, 1998. With additional material by M. Joshi.

[FS14] R.L. Frank and B. Schlein. Dynamics of a strongly coupled polaron. Lett. Math. Phys., 104(8):911-929, 2014.

[FTY02] Jürg Fröhlich, Tai-Peng Tsai, and Horng-Tzer Yau. On the point-particle (Newtonian) limit of the non-linear Hartree equation. Comm. Math. Phys., 225(2):223-274, 2002.

[Lie77] Elliott H. Lieb. Existence and uniqueness of the minimizing solution 
of Choquard's nonlinear equation. Studies in Appl. Math., 57(2):93-105, 1976/77.

[MZ10] Li Ma and Lin Zhao. Classification of positive solitary solutions of the nonlinear Choquard equation. Arch. Ration. Mech. Anal., 195(2):455-467, 2010.

[Paz83] A. Pazy. Semigroups of linear operators and applications to partial differential equations, volume 44 of Applied Mathematical Sciences. Springer-Verlag, New York, 1983.

[Pen98] Quantum computation, entanglement and state reduction. Philosophical Transactions of the Royal Society of London A: Mathematical, Physical and Engineering Sciences, 356(1743):1927-1939, 1998.

[Sch95] G. Schneider. Validity and limitation of the Newell-Whitehead equation. Math. Nachr., 176:249-263, 1995.

[SSZ15] G. Schneider, D.A. Sunny, and D. Zimmermann. The NLS approximation makes wrong predictions for the water wave problem in case of small surface tension and spatially periodic boundary conditions. Journal of Dynamics and Differential Equations, 27(3):1077-1099, 2015. 